\title{
Isotopic equilibrium constants for very low-density and low-temperature nuclear matter
}

\author{
J. B. Natowitz ${ }^{1}$, H. Pais ${ }^{2}$, G. Röpke ${ }^{3}$, J. Gauthier ${ }^{1}$, K. Hagel ${ }^{1}$, M. Barbui ${ }^{1}$, and R. Wada ${ }^{1}$ \\ ${ }^{1}$ Cyclotron Institute, Texas A\&M University, College Station, Texas 77843 \\ ${ }^{2}$ Department of Physics, University of Coimbra, 3004-516 Coimbra, Portugal and \\ ${ }^{3}$ University of Rostock, FB Physik, 18059 Rostock, Germany
}

(Dated: September 14, 2020)

\begin{abstract}
Yields of equatorially emitted light isotopes, $1 \leq Z \leq 14$, observed in ternary fission in the reaction ${ }^{241} \mathrm{Pu}\left(n_{\mathrm{th}}, \mathrm{f}\right)$ are employed to determine apparent chemical equilibrium constants for lowtemperature and low-density nuclear matter. The degree of equilibration and role of medium modifications are probed through a comparison of experimentally derived reaction quotients with equilibrium constants calculated using a relativistic mean-field model employing a universal medium modification correction for the attractive $\sigma$ meson coupling. The results of these comparisons indicate that equilibrium is achieved for the lighter ternary fission isotopes. For the heavier isotopes experimental reaction quotients are well below calculated equilibrium constants. This is attributed to a dynamical limitation reflecting insufficient time for full equilibrium to develop. The role of medium effects leading to yield reductions is discussed as is the apparent enhancement of yields for ${ }^{8} \mathrm{He}$ and other very neutron rich exotic nuclei.
\end{abstract}

\section{INTRODUCTION}

A high quality nuclear equation of state (EOS) applicable over a wide range of density and temperature is an essential ingredient for reliable simulations of stellar matter and astrophysical phenomena. In recent decades many nuclear theory efforts have been devoted to developing such equations and many are available in the literature, see Refs. [1-14] and references therein. The validation of these equations of state usually rests on careful comparisons between the results of theoretical simulations and astrophysical observations.

At the same time, laboratory studies of nuclear matter at different densities, temperatures and isospin content offer some unique possibilities to address specific aspects of the nuclear equation of state. Exploiting a variety of projectile energies, projectile-target combinations and reaction mechanisms, nuclear experimentalists have probed cluster formation and the composition of nuclear matter at different densities, caloric curves and phase transitions, the density dependence of the symmetry energy and medium effects on nuclear binding energies, see Refs. [12 19] and references therein.

While isotope mass fractions are commonly used to present the results of EOS composition calculations, references 13, 14, 18, 19] employed chemical equilibrium constants for production of $Z=1(\mathrm{H})$ and $Z=2(\mathrm{He})$ derived from the experimental isotope yields. These are more robust quantities for testing different equations of state since, at least in the low-density ideal limit, they are less dependent upon the choice of isotopes included in the EOS model calculations and upon the source asymmetry.

The thermodynamic reaction quotient $Q$ for the formation of an isotope ${ }^{A} Z$ with mass number $A$, atomic number $Z$, and neutron number $N=A-Z$, is defined such that,

$$
Q=\frac{\left\{{ }^{A} Z\right\}}{\{p\}^{Z}\{n\}^{N}}
$$

where curly brackets denote the fugacities of the chemical species, i.e. the isotope ${ }^{A} Z$ as well as the protons $(p)$ and neutrons $(n)$. Fugacity depends on temperature, pressure and composition of the mixture, among other things. The fomulation in terms of fugacities arises because components in non-ideal systems interact with each other. In nuclear EOS models these interactions are modeled in a variety of ways [1, 2, 4, 6 11, 13, 14, 19]. The right-hand side of this equation corresponds to the reaction quotient for arbitrary values of the fugacities. The reaction quotient becomes the equilibrium constant, $K$, if the system reaches equilibrium. The equilibrium constant is related to the standard Gibbs free energy change for the reaction, $\Delta G^{0}$, as

$$
\Delta G^{0}=-R T \ln K
$$

where $T$ is the temperature and $R$ is the gas constant.

If deviations from ideal behavior are neglected, the fugacities may be replaced by concentrations or densities. Employing square brackets to indicate concentrations or densities at equilibrium we can designate this ratio as chemical constant $K_{c}$,

$$
K_{c}=\frac{\left[{ }^{A} Z\right]}{[p]^{Z}[n]^{N}} .
$$

$K_{c}$ is defined in an equivalent way to the thermodynamic equilibrium constant but with concentrations or densities of reactants and products, denoted by square brackets, instead of fugacities.

The experimental equilibrium constants reported in references [18, 19] demonstrated clearly that, even at densities in the 0.003 to 0.03 nucleons $/ \mathrm{fm}^{3}$ range, interactions are important and experimental equilibrium 
constant data may be employed to evaluate the various theoretical models.

\section{ANALYSIS OF TERNARY FISSION YIELDS}

In this paper we report extensions of the measurements of isotopic equilibrium constants to a broader range of isotopes at even lower temperature and densities. Specifically we derive isotopic equilibrium constants for isotopes produced in ternary fission processes which occur in approximately $0.3 \%$ of decays during the spontaneous or thermal neutron induced fission of a heavy nucleus $20-$ 45]. Such ternary fission is characterized by emission of an energetic light particle or fragment in a direction perpendicular to the axis defined by the separating massive fragments, signaling their origin in the region between the two nascent heavy fragments at or near the time of scission. Collectively, such isotopes, are typically identified in the ternary fission literature as "scission" or "equatorially" emitted particles.

This well identified isolated mechanism facilitates exploration of yields with minimal perturbations from collision dynamics. This allows an experimental test of the chemical equilibrium hypothesis. If that hypothesis is supported, derived equilibrium constants provide information against which various proposed equations of state may be tested in the low-density limit. In this regard they constitute the experimental counterpart of theoretical virial equations of state which serve as a low-density theoretical baseline for EOS calculations [4, 10]. Data of sufficient accuracy would allow a careful evaluation of the density dependence of fragment-fragment interactions and in medium modifications of cluster properties. See reference [46] for a recent discussion of such effects.

The experimental results of Koester et al., obtained with an on-line mass spectrometer, provide a comprehensive data set for ternary fission yields for 42 isotopes determined in the reaction ${ }^{241} \mathrm{Pu}\left(n_{\mathrm{th}}, \mathrm{f}\right)$ [28, 29]. In addition, 17 upper limits are also reported for yields of other isotopes. In reference [45], these yields were compared to results of calculations made using a model which assumes a nucleation-time-moderated chemical equilibrium [47 49] in the low-density matter which constitutes the neck region of the scissioning system. Nucleation approaches have much in common with thermal coalescence approaches previously applied to clustering in lowdensity nuclear systems [50, 51] but explicitly incorporate consideration of cluster formation rates. Coalescence of nucleons into clusters is a dynamic process requiring time, while the fissioning system exists for a limited time span. A reasonably good fit to the ${ }^{241} \mathrm{Pu}\left(n_{\mathrm{th}}, \mathrm{f}\right)$ experimental data from references [28, 29] was obtained with the following parameters: Temperature, $T=1.4 \mathrm{MeV}$, density, $\rho=4 \times 10^{-4} \mathrm{fm}^{-3}$, proton fraction, $Y_{p}=0.34$, nucleation time $t_{\text {nuc }}=6400 \mathrm{fm} / c$ and critical cluster mass, $A_{\text {cr }}=5.4$. We note that various previous attempts to evaluate the temperatures appropriate to thermal neu- tron induced ternary fission have led to temperatures in the range of 1.0 to $1.4 \mathrm{MeV}$ 52, 53]. For the ${ }^{242} \mathrm{Pu}$ compound nucleus the proton fraction, $Y_{p}$, is 0.388 . The derived value of 0.34 indicates that the region between the separating fragments, which dominates the production of the ternary particles, is neutron enriched [45, 54].

\section{EQUILIBRIUM CONSTANT FOR ${ }^{4} \mathrm{He}$}

Since all yields in the Koester ${ }^{241} \mathrm{Pu}\left(n_{\mathrm{th}}, \mathrm{f}\right)$ data are referenced to the yield of ${ }^{4} \mathrm{He}$ particles we began by establishing the correspondent equilibrium constant for this particle. Determining this equilibrium constant requires accurate yields of the neutrons, protons and ${ }^{4} \mathrm{He}$ ejected at the time of scission. The equatorial emission origin of these particles must be well defined and contributions from other sources (e.g. pre-scission emission, polar emission, secondary particle emission) to the total yields be carefully removed. Establishing the yields of equatorial emission requires careful exploration of the particle angular distributions relative to the scission axis. These measurements are difficult, particularly for the neutrons because subsequent evaporation from the fission fragments dominates the neutron yield. Fortunately, very precise measurements of these yields have been made by a number of extremely competent experimental groups and absolute yields for many fissioning isotopes are, in fact, available and tabulated in the literature [20 44]. The systematics of ternary fission yields have been extensively analyzed in various evaluations and review articles $[2022,26]$. Focusing particularly on values reported for $\mathrm{Pu}$ isotopes we have adopted for our calculations the experimental values indicated in column 4 of Table I. The adopted value for the ${ }^{4} \mathrm{He}$ particle yield includes a $17 \%$ correction to remove ${ }^{4} \mathrm{He}$ particles resulting from the decay of ${ }^{5} \mathrm{He}$ nuclei emitted at scission [37]. The adopted value for protons includes a $14.5 \%$ correction to remove polar emission protons 39 41]. For neutrons, the adopted value is that determined for scission neutrons [36].

Applying the thermal coalescence model of Mekjian [50] to these data allows extraction of the coalescence volume, $2937 \mathrm{fm}^{3}$. With the absolute yields and this coalescence volume, the relevant densities and the experimental equilibrium constant $K_{c}\left({ }^{4} \mathrm{He}\right)$ for direct formation of the ${ }^{4} \mathrm{He}$ in its ground state is $3.02( \pm 1.07) \times 10^{18} \mathrm{fm}^{9}$. As indicated above, the largest contributor to the uncertainty is the neutron scission yield. Note, however, that the apparent effective $K_{c}^{\text {eff }}$ for the total experimentally observed ${ }^{4} \mathrm{He}$ yield (column 2, Table】), which includes the ${ }^{5} \mathrm{He}$ contribution (as well as possible smaller contributions from other particle unstable isotopes) is $3.66( \pm 1.30) \times 10^{18} \mathrm{fm}^{9}$. By convention, relative yields in ternary fission are typically normalized to the total ${ }^{4} \mathrm{He}$ yield.

In reference [13] Pais et al. reported a study of inmedium modifications on light cluster properties, within 


\begin{tabular}{|c|c|c|c|}
\hline particle & total yield/fission & equatorial scission emission & adopted yield \\
\hline$n$ & $2.96 \pm 0.005$ & $0.107 \pm 0.015$ & $0.107 \pm 0.015$ \\
$p$ & $4.08 \times 10^{-5} \pm 0.41$ & $3.49 \times 10^{-5} \pm 0.35$ & $3.49 \times 10^{-5} \pm 0.35$ \\
${ }^{4} \mathrm{He}$ & $2.015 \times 10^{-3} \pm 0.20$ & $2.00 \times 10^{-3} \pm 0.20$ & $1.66 \times 10^{-3} \pm 0.17$ \\
\hline
\end{tabular}

TABLE I: Adopted values of neutron, proton and ${ }^{4}$ He yields [21, 22, 35 44]. References are the primary sources. Measurements and systematics of other data for adjacent isotopes were also employed in establishing these values. Uncertainties are $1 \sigma$.

the relativistic mean-field approximation, where explicit binding energy shifts and a modification on the scalar cluster-meson coupling were introduced in order to take these medium effects into account. The interactions of the clusters with the surrounding medium are described with a phenomenological modification, $x_{i, \sigma}$, of the coupling constant to the $\sigma$ meson, $g_{i, \sigma}=x_{i, \sigma} A_{i} g_{\sigma}$. Using the FSU Gold EOS [12] and requiring that the cluster fractions exhibit the correct behavior in the low-density virial limit [4, 9, 10], they obtained a universal scalar clustermeson coupling fraction, $x_{i, \sigma}=0.85 \pm 0.05$, which could reproduce both this limit and the equilibrium constants extracted from reaction ion data [18, 19] reasonably well. The results are qualitatively similar to the ones obtained with other approaches $[4,6$, 9, 19]. Employing the model of reference [13] with $T=1.4 \mathrm{MeV}, \rho_{\text {tot }}=4 \times 10^{-4} \mathrm{fm}^{-3}$, and a scalar cluster-meson coupling fraction $x_{i, \sigma}=0.85$ leads to $K_{c}\left({ }^{4} \mathrm{He}\right)=2.99 \times 10^{18} \mathrm{fm}^{9}$ for direct production, and $K_{c}^{\text {eff }}\left({ }^{4} \mathrm{He}\right)=3.65 \times 10^{18} \mathrm{fm}^{9}$.

In a more recent work 14, Pais et al. compared their model results to equilibrium constants calculated from a new analysis, where in-medium modifications are addressed, for experimental data measured in intermediate energy $\mathrm{Xe}+\mathrm{Sn}$ collisions. This comparison lead to a higher scalar cluster-meson coupling constant $x_{i, \sigma}=0.92 \pm 0.02$.

With this higher assumed value of the coupling constant, the in-medium effects are reduced, and the predicted value for $K_{c}\left({ }^{4} \mathrm{He}\right)$ becomes $3.75 \times 10^{18} \mathrm{fm}^{9}$, and for $K_{c}^{\text {eff }}\left({ }^{4} \mathrm{He}\right)=4.62 \times 10^{18} \mathrm{fm}^{9}$.

\section{EXTENSION TO OTHER ISOTOPES}

Using the adopted values of the equatorial neutron and proton yields together with the measured yields for all isotopes we have calculated the effective experimental reaction quotients, $Q_{c}^{\text {eff }}$ for formation of the observed isotopes from the nucleons, i. e.,

$$
Q_{c}^{\mathrm{eff}}=\frac{\left[{ }^{A} Z\right]}{[p]^{Z}[n]^{N}}
$$

where eff denotes total observed yields including all contributions from gamma decaying and particle decaying excited states. Here we employ $Q$ because in our previous treatment of these same data within the framework of a nucleation time modulated statistical equilibrium model we have presented evidence that statistical equilibrium is not achieved for the heaviest isotopes 45]. The term effective is used in recognition of the fact that the final observed ground state yields include contributions from de-excitation of short lived gamma or particle decaying states initially present in the primary isotope distribution. The relative importance of such contributions will vary with temperature and density. For a system at equilibrium $Q_{c}^{\text {eff }}=K_{c}^{\text {eff }}$, the effective equilibrium constant. A direct comparison between the experimental results and those of theoretical calculations requires that the contributions from relevant excited states be included in the theoretical treatment.

In the original formulation by Pais et al., only ground states including particle unstable ground states were included in the calculation. For the present calculation we have included experimentally identified (excitation energy and spin) gamma decaying excited states 57. which can have a significant population at $T=1.4 \mathrm{MeV}$. We have also included relevant particle unstable isotopes and states which can feed the observed population [57]. With this ensemble of states we performed some preliminary calculations to explore the sensitivity of various results to the assumed density. We found the final free neutron to free proton ratio to be very sensitive to density. A comparison of the theoretical free $n / p$ ratio to the experimentally observed free $n / p$ ratio for different assumed total densities indicated a density of $2.56( \pm 0.20) \times 10^{-4} \mathrm{fm}^{-3}$. This value, which is somewhat lower than the $4 \times 10^{-4} \mathrm{fm}^{-3}$ derived from nucleation model fits, has been adopted for the present calculations. In the recent treatment of the emisssion of $Z=1,2$ isotopes in the spontaneous ternary fission of ${ }^{252} \mathrm{Cf}$ a different approach suggests quite similar values [46].

The experimentally derived reaction quotients are presented in Figure 1. To more clearly present the data, we plot $Q_{c}^{\text {eff }}$ against the isotope identifier parameter proposed by Lestone [30], i.e., $A+8(Z-1)$. For comparison to the experimental $Q_{c}^{\text {eff }}$ values, we also present theoretically calculated equilibrium constants, $K_{c}^{\text {eff }}$, obtained using the model of Pais et al. [13] with a scalar clustermeson coupling constant $x_{i, \sigma}$ of 0.92 . To carry out these calculations we fixed the temperature to be $1.4 \mathrm{MeV}$, the total density to be $2.56 \times 10^{-4}$ nucleons $/ \mathrm{fm}^{3}$ and the proton fraction of the matter to be 0.34 . Both the experimental and theoretical values are tabulated in Appendix A of this paper. Unlike the data employed for the previous comparisons with this model, the present data include isotopes as heavy as ${ }^{36} \mathrm{Si}$. Therefore the role of excited states should be much more important in 


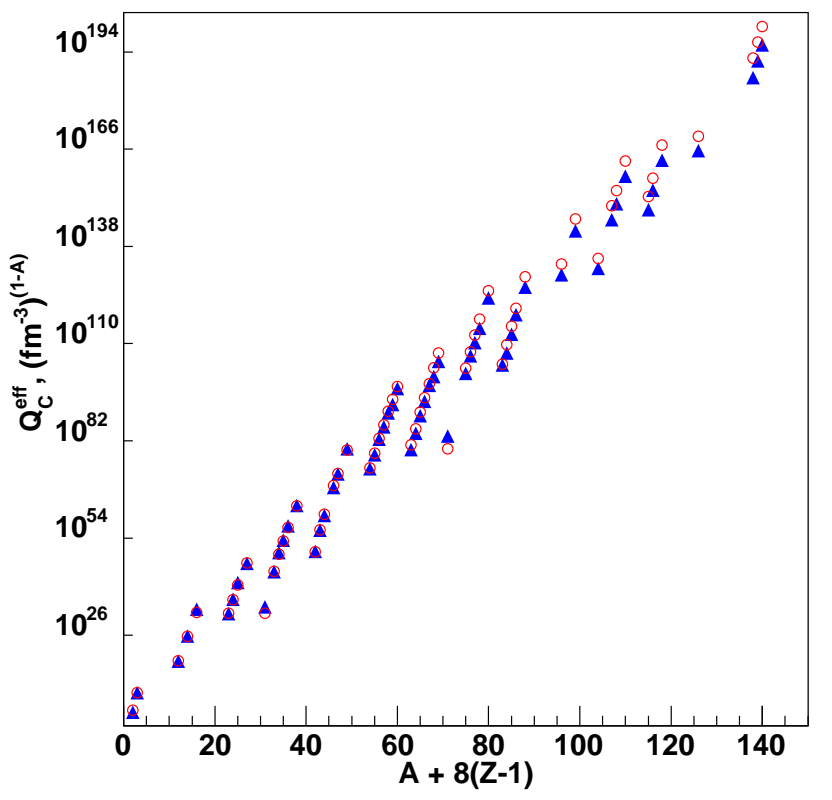

FIG. 1: $Q_{c}^{\text {eff }}$ values vs $A+8(Z-1)$. Triangles - experimental results. Open circles - theoretical results for $K_{c}^{\text {eff }}$ with $T=1.4$ $\mathrm{MeV}, Y_{p}=0.34, \rho=2.56 \times 10^{-4} \mathrm{fm}^{-3}$ and coupling constant $x_{i, \sigma}=0.92$.

determining the observed isotope yields. This is particularly true for nuclei with lower energy gamma decaying excited states with high degeneracies. Particle decaying excited states are also included but many generally occur at relatively higher excitation energies and thus are less populated at low temperature.

As is observed in Figure 1, the experimental and theoretical trends are quite similar. For the heaviest isotopes there is, however a clear indication that the experimental $Q_{c}^{\text {eff }}$ values fall well below the theoretically calculated equilibrium constants. To better appreciate these differences we plot, in Figure 2 the ratios of the values of the experimentally derived reaction coefficients to the $K_{c}^{\text {eff }}$ values calculated theoretically using the Pais et al. formulation [13]. Ratios for isotopes for which measured experimental yield values exist are identified by triangles. Those for which only upper limits to the experimental yields are available are not included in this figure.

In Figure 2 we see that for the lighter isotopes there is some scatter about the ratio $R_{\exp / \text { theo }}=Q_{c}^{\exp } / Q_{c}^{\text {theo }}=1$, but a general overall accord between the data and the theoretical values, suggesting that chemical equilibrium has been achieved for the isotopes with $Z \leq 5$. The experimental $K_{c}$ value reported for the ${ }^{2} \mathrm{H}$ is well below the theoretical value. This appears to reflect the very weak binding of the deuteron. Such reductions in deuteron yield are a general feature in the production of deuterons in heavy ion collisions $13,18,56$. Interestingly, for the light neutron rich isotopes ${ }^{8} \mathrm{He},{ }^{9} \mathrm{Li},{ }^{10} \mathrm{Be}$ and ${ }^{12} \mathrm{Be}$ the

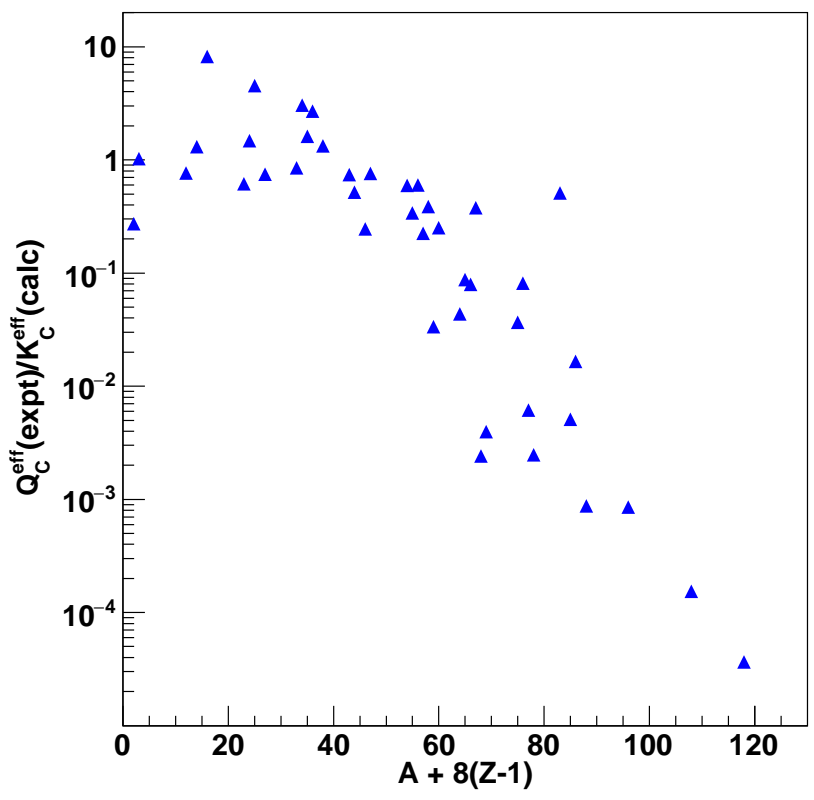

FIG. 2: Ratio $Q_{c}^{\mathrm{eff}}$ (experiment) $/ Q_{c}^{\mathrm{eff}}$ (theory) vs $A+8(Z-1)$. All isotopes in Koester data table are considered. Isotopes for which only upper limits are reported are excluded from this plot. See text.

ratio indicates significant excesses relative to the calculated values in the region where there is a reasonable agreement for the other isotopes.

Above that point the plotted ratios drop rapidly falling to $R_{\exp / \text { theo }} \sim 10^{-5}$ for the heaviest isotopes. Since the Pais calculation includes medium effects through the cluster coupling constant this decrease does not appear to reflect calculated medium effects. Rather, the observed decline in the ratio of experimental value to theoretical value indicates that equilibrium is not reached for the heavier isotopes. This is entirely consistent with the conclusion reached in reference [45] where it is attributed to a time moderated nucleation effect. The possibility that finite size effects may also contribute to this decline is not ruled out.

\section{V. $Z=1$ AND 2 ISOTOPES AND MEDIUM MODIFICATIONS}

Given the recent detailed analysis of $Z=1(\mathrm{H})$ and 2 (He) isotope production for ${ }^{252} \mathrm{Cf}$ ternary fission [46] it is interesting to focus explicitly on these results for the present case. In Table II the available measured equilibrium constants for these isotopes are presented and compared to the theoretical values calculated using a scaler cluster-meson coupling constant $x_{i \sigma}=0.92$. As already noted above, the experimental $Q_{c}$ value based on the observed yield for the ${ }^{2} \mathrm{H}$ is well below the theo- 


\begin{tabular}{|c|c|c|}
\hline particle & $Q_{c}^{\text {eff }}($ expt $)$ & $K_{c}^{\text {eff }}($ calc $)$ \\
\hline${ }^{2} \mathrm{H}$ & $5.50( \pm 0.99) \times 10^{3}$ & $2.42 \times 10^{4}$ \\
${ }^{3} \mathrm{H}$ & $2.84( \pm 0.85) \times 10^{9}$ & $3.29 \times 10^{9}$ \\
${ }^{3} \mathrm{He}$ & & $1.43 \times 10^{9}$ \\
${ }^{4} \mathrm{He}$ & $3.66( \pm 1.30) \times 10^{18}$ & $4.74 \times 10^{18}$ \\
${ }^{5} \mathrm{He}$ & & $1.50 \times 10^{22}$ \\
${ }^{6} \mathrm{He}$ & $5.95( \pm 3.50) \times 10^{25}$ & $5.45 \times 10^{25}$ \\
${ }^{7} \mathrm{He}$ & & $2.60 \times 10^{28}$ \\
${ }^{8} \mathrm{He}$ & $2.60( \pm 1.97) \times 10^{33}$ & $3.76 \times 10^{32}$ \\
\hline
\end{tabular}

TABLE II: Chemical constants for the isotopes of the light elements H, He. The experimental values $Q_{c}^{\text {eff }}$ (expt) are compared to calculated values $K_{c}^{\text {eff }}$ (calc).

retical value. (This is also true in the ${ }^{252} \mathrm{Cf}$ case [46].) This suggests a clear medium effect for this very weakly bound nucleus [55, 58]. ${ }^{3} \mathrm{He}$ was not observed in the Koester experiment nor has a ${ }^{3} \mathrm{He}$ yield been reported in any other ternary fission experiment [37, 38]. The theoretically calculated ${ }^{3} \mathrm{He}$ and ${ }^{3} \mathrm{H}$ equilibrium constants in Table II are similar, as expected, the difference arising from the small binding energy difference for these $A=3$ isotopes. While some similar medium effect may operate on the $A=3$ yields, the non-observation of ${ }^{3} \mathrm{He}$ reflects the very small free proton to free neutron ratio at equilibrium indicated in Table 1 . Given that ratio, the ${ }^{3} \mathrm{He}$ yield should be about four orders of magnitude below the ${ }^{3} \mathrm{H}$ yield. This low yield, together with possible additional factors specific to individual experiments. e.g., separation, identification and background discrimination, offers a natural explanation for the absence of ${ }^{3} \mathrm{He}$ yield data in the literature.

For ${ }^{3} \mathrm{H},{ }^{4} \mathrm{He}$ and ${ }^{6} \mathrm{He}$ the tabulation indicates reasonable agreement (within experimental errors) between experiment and theory.

In contrast the experimental value for the very neutron rich ${ }^{8} \mathrm{He}$ is an order of magnitude higher than that calculated. The large experimental yield of ${ }^{8} \mathrm{He}$ is a general feature of ternary fission experiments. This special nature of ${ }^{8} \mathrm{He}$ may reflect some feature of dynamics, e.g., time dependent density or temperature fluctuations or feeding from parent nuclei, or of detailed structural features not yet understood. As noted in the previous section the comparison of the experimental equilibrium constants with those of the calculation (Figures 1. 2. Tables IV(a), IV(b) also indicates yield enhancements for the other neutron rich isotopes ${ }^{9} \mathrm{Li},{ }^{10} \mathrm{Be}$ and ${ }^{12} \mathrm{Be}$. The cluster structure of such neutron rich nuclei has been discussed in the framework of an extended Ikeda diagram [59]. Particularly intriguing is the possibility that the yield enhancement reflects the existence of strong neutron correlations in the disassembling matter. In this regard ${ }^{8} \mathrm{He}$ is of special interest as experimental evidence for a possible alpha-tetra-neutron structure has been published [60] and some theoretical work suggests that a tetra-neutron condensate might be formed in low-density neutron rich stellar matter [61]. This subject warrants further investigation.

In a recent related paper on the spontaneous ternary fission of ${ }^{252} \mathrm{Cf}$ [46], we explored an alternative information entropy based analysis to characterize the emission of $Z=1,2$ isotopes as a basis for evaluating medium effects. In that paper it was proposed that relevant primary distribution of isotopes formed in the ternary fission process could be characterized by a few Lagrange parameters $\lambda_{T}, \lambda_{n}, \lambda_{p}$ such that

$Y_{A, Z}^{\mathrm{rel}} \propto R_{A, Z}^{\mathrm{rel}} g_{A, Z}\left(\frac{2 \pi \hbar^{2}}{A m \lambda_{T}}\right)^{-\frac{3}{2}} e^{\left[B_{A, Z}+(A-Z) \lambda_{n}+Z \lambda_{p}\right] / \lambda_{T}}$

where $g_{A, Z}$ denotes the degeneracy of the nucleus $\{A, Z\}$ in the ground state, $B_{A, Z}$ its binding energy, $m$ is the average nucleon mass. The Lagrange parameters $\lambda_{i}$ are non-equilibrium generalizations of the equilibrium thermodynamic parameters $T, \mu_{n}, \mu_{p}$. Different approximations to treat the Hamiltonian of the many-nucleon system lead to different values for these parameters. In particular all relevant excited states and continuum states have to be taken into account, and in-medium mean-field and Pauli blocking effects must be included. These effects are collected in a prefactor $R_{A, Z}^{\mathrm{rel}}$ which, in general, depends on the Lagrange parameters $\lambda_{i}$.

The relevant primary isotopic distribution is related to the observed distribution via a non-equilibrium evolution, which is described in simplest approximation by reaction kinetics where unstable nuclei feed the observed yields of stable nuclei. As detailed in Ref. [46], taking into account all bound states below the edge of continuum states, the primary distribution $Y_{A, Z}^{\mathrm{rel}, \gamma}$ can be obtained, with Lagrange parameters $\lambda_{i}^{\gamma}$ obtained from a least squares fit to the observed final yields of ${ }^{2} \mathrm{H},{ }^{3} \mathrm{H}$, ${ }^{4} \mathrm{He},{ }^{6} \mathrm{He}$, and ${ }^{8} \mathrm{He}$. The correct treatment of continuum states gives the virial expansion which is exact in the lowdensity limit. Using measured scattering phase shifts, virial expansions have been determined for ${ }^{2} \mathrm{H},{ }^{4} \mathrm{H},{ }^{5} \mathrm{He}$ and ${ }^{8} \mathrm{Be}$ (which feeds ${ }^{4} \mathrm{He}$ ), see Ref. [4, 62]. For the other isotopes estimates are given in [46]. Such a treatment including the continuum states leads to a significant reduction in the calculated yields of the unbound nuclei ${ }^{4} \mathrm{He}$, ${ }^{5} \mathrm{He},{ }^{7} \mathrm{He}$, and ${ }^{9} \mathrm{He}$. As was shown in the ternary spontaneous fission of ${ }^{252} \mathrm{Cf}$ [46], the observed yield of ${ }^{6} \mathrm{He}$ is overestimated, and the observed yield of ${ }^{8} \mathrm{He}$ is underestimated. A possible explanation may be in-medium corrections, in particular Pauli blocking. ${ }^{6} \mathrm{He}$ is only weakly bound (the edge of continuum states is at $0.975 \mathrm{MeV}$ which is small compared even to $2.225 \mathrm{MeV}$ for ${ }^{2} \mathrm{H}$ ) so that Pauli blocking may dissolve the bound state at increasing density. To reproduce the observed yields, we have determined an effective pre-factor $R_{A, Z}^{\mathrm{rel}, \mathrm{eff}}$. Both, the effective pre-factor and the relevant primary yields required to reproduce the observed yields are shown in Table III] In detail, the pre-factor $R_{A, Z}^{\text {rel,eff }}$ which represents the internal partition function was taken from the virial expansion for ${ }^{2} \mathrm{H},{ }^{3} \mathrm{H},{ }^{3} \mathrm{He},{ }^{4} \mathrm{He},{ }^{5} \mathrm{He},{ }^{8} \mathrm{Be}$, as well as the 
estimates for ${ }^{8} \mathrm{He}$ and ${ }^{9} \mathrm{He}$. The corresponding observed yields are used to determine the three Lagrange parameters $\lambda_{T}, \lambda_{n}, \lambda_{p}$. To reproduce the observed (weakly bound) ${ }^{6} \mathrm{He}$, the effective factor $R_{6}^{\mathrm{rel}} \mathrm{He}$ was determined. For this, the contribution of the primary yields of ${ }^{6} \mathrm{He}$ and ${ }^{7} \mathrm{He}$ must be known. We used the value $Y^{7} \mathrm{He} / Y_{6} \mathrm{He}=0.21$ measured for ${ }^{252} \mathrm{Cf}$ in [35]. It would be of interest to verify these predictions of $Y_{A, Z}^{\text {rel,eff }}$ by measurements for ${ }^{242} \mathrm{Pu}$ as were done for ${ }^{252} \mathrm{Cf}$.

Interpreting the effective pre-factors $R_{A, Z}^{\text {rel,eff }}$ as reflecting in-medium corrections, we can use these inferred values to estimate the density. These in-medium corrections are single-nucleon self-energy shifts which may be absorbed into the Lagrange parameters $\lambda_{n}, \lambda_{p}$ if momentum dependence of the single-particle self-energy is neglected. Then, the density dependence of $R_{A, Z}^{\text {rel,eff }}$ is governed by the Pauli blocking effects which reduce the binding energies. A global reduction of the binding energies is described in the generalized RMF approximation $\left(x_{i, \sigma}=0.92\right)$ given above by the effective cluster coupling to the mesonic field. Within a more individual calculation, the Pauli blocking acts stronger for weakly bound states, eventually dissolving them, denoted as the Mott effect [55, 58]. We have performed an exploratory calculation assuming that Pauli blocking is essential for ${ }^{6} \mathrm{He}$ because of its small binding but neglect the Pauli blocking shift for the stronger bound nuclei. The reduction factor $R_{6 \mathrm{He}}^{\mathrm{rel}, \text { eff }}$ derived for ${ }^{6} \mathrm{He}$ is smaller than the expected value $R_{6 \mathrm{He}}^{\mathrm{rel}, \mathrm{vir}}=0.945$ according to the virial expansion. This leads to a shift of the binding energy of about $0.9 \mathrm{MeV}$ and a correspondent density value of about $n_{n}=0.0006$ $\mathrm{fm}^{-3}$. Note that this value has a large error because of uncertainties in the observed yield of ${ }^{6} \mathrm{He}$ as well as the estimation of the energy shift of ${ }^{6} \mathrm{He}$ due to in-medium corrections. Large deviations from the simple NSE are predicted for the primary yields of ${ }^{5} \mathrm{He}$, and it would be of interest to observe it like in the case of ${ }^{252} \mathrm{Cf}$ [46].

A paper in which this approach is followed more consistently, considering also the Pauli blocking shifts for strongly-bound nuclei, and calculating the ternary fission yields for the $Z=3-14$ isotopes observed in ${ }^{241} \mathrm{Pu}\left(n_{\mathrm{th}}, \mathrm{f}\right)[28,29]$ is currently in preparation [63].

\section{SUMMARY AND DISCUSSION}

In conclusion, experimentally determined reaction quotients have been determined for equatorially ejected isotopes of $Z \leq 14$ observed in the ternary fission of ${ }^{242} \mathrm{Pu}$. The emission is characterized by $T=1.4 \mathrm{MeV}, Y_{p}=0.34$ and $\rho=2.6 \times 10^{-4}$ nucleons $/ \mathrm{fm}^{3}$. It should be noted that since at equilibrium the reaction coefficients are primarily sensitive to temperature and to density through medium effects extraction of accurate densities remains a difficult problem. Here we have used the observed free neutron to free proton ratio to establish the density.

A comparison of the reaction quotients with those cal-

\begin{tabular}{|c|c|c|c|c|c|}
\hline isotope & $Y_{A, Z}^{\text {exp }}$ & $\frac{B_{A, Z}}{A}[\mathrm{MeV}]$ & $g_{A, Z}$ & $R_{A, Z}^{\text {rel,eff }}$ & $Y_{A, Z}^{\text {rel,eff }}$ \\
\hline$\lambda_{T}$ & - & - & - & - & 1.2042 \\
$\lambda_{n}$ & - & - & - & - & -2.9954 \\
$\lambda_{p}$ & - & - & - & - & -16.633 \\
\hline${ }^{1} \mathrm{n}$ & - & 0 & 2 & - & 1588200 \\
${ }^{1} \mathrm{H}$ & - & 0 & 2 & - & 19.16 \\
${ }^{2} \mathrm{H}$ & 42 & 1.112 & 3 & 0.98 & 42 \\
${ }^{3} \mathrm{H}^{\text {obs }}$ & 786 & 2.827 & 2 & - & 786 \\
${ }^{3} \mathrm{H}$ & - & 2.827 & 2 & 0.99 & 779.51 \\
${ }^{4} \mathrm{H}$ & - & 1.720 & 5 & 0.0606 & 6.46679 \\
${ }^{3} \mathrm{He}$ & - & 2.573 & 2 & 0.988 & 0.004972 \\
${ }^{4} \mathrm{He} \mathrm{e}^{\text {obs }}$ & 10000 & 7.073 & 1 & - & 10000 \\
${ }^{4} \mathrm{He}$ & - & 7.073 & 1 & 1 & 8485.89 \\
${ }^{5} \mathrm{He}$ & - & 5.512 & 4 & 0.7028 & 1508.81 \\
${ }^{6} \mathrm{He}$ & 260 & 4.878 & 1 & - & 260 \\
${ }^{6} \mathrm{He}$ & - & 4.878 & 1 & 0.8827 & 14.868 \\
${ }^{7} \mathrm{He}$ & - & 4.123 & 4 & 0.6235 & 45.122 \\
${ }^{8} \mathrm{He} \mathrm{e}^{\text {obs }}$ & 15 & 3.925 & 1 & - & 15 \\
${ }^{8} \mathrm{He}$ & - & 3.925 & 1 & 0.9783 & 14.72 \\
${ }^{9} \mathrm{He}$ & - & 3.349 & 2 & 0.2604 & 0.27 \\
${ }^{8} \mathrm{Be}-$ & - & 7.062 & 1 & 1.07 & 2.65 \\
\hline
\end{tabular}

TABLE III: Properties and relative yields of the $\mathrm{H}, \mathrm{He}$ and $\mathrm{Be}$ isotopes from ternary fission ${ }^{241} \mathrm{Pu}\left(n_{\mathrm{th}}, \mathrm{f}\right)$ which are relevant for the observed yields of $\mathrm{H}$, He nuclei, (denoted by the superscript 'obs'.) Experimental yields $Y_{A, Z}^{\exp }$ [29] are compared to the yields calculated as described in the text. Observed yields - column 2 , binding energy $B_{A, Z} / A$ - column 3 , ground state degeneracy - column 4. prefactor - column 5 , calculated primary isotope distribution - column 6 .

culated using the EOS model of Pais et al. [13] with a scaler cluster-meson coupling constant of $x_{i, \sigma}=0.92$ indicates a reasonable agreement between the experimental results and the model calculations for the lighter isotopes, indicating that chemical equilibrium is achieved for those isotopes and that medium effects are quite small at this temperature and density. A more detailed evaluation of possible medium effects at these densities, addressing the properties of individual isotopes, is presented in reference [46]. The experimental yield of ${ }^{8} \mathrm{He}$ is much higher than predicted in the calculation. Other very neutron rich isotopes, ${ }^{9} \mathrm{Li},{ }^{10} \mathrm{Be}$ and ${ }^{12} \mathrm{Be}$, also give evidence of being underestimated in the calculation. Whether this reflects the particular structural characteristics of these exotic nuclei warrants careful investigation [60, 61]. For the heavier isotopes, the ratio of the measured reaction coefficient to the theoretically predicted equilibrium constant exponentially decreases with increasing mass. This is attributed to a dynamical limitation, reflecting insufficient time for full equilibrium to develop [45]. An important point to be emphasized is that valid comparisons of calculated equilibrium constants to those derived from experimental data demand that the actual experimental ensemble of competing species be replicated as fully as 


\begin{tabular}{|c|c|c|c|}
\hline isotope & $Q_{c}^{\text {eff }}$ expt. & $Q_{c}^{\text {eff }}$ calc. & upper limit \\
\hline${ }^{2} \mathrm{H}$ & $6.61 \mathrm{E}+03$ & $2.42 \mathrm{E}+04$ & \\
${ }^{3} \mathrm{H}$ & $3.39 \mathrm{E}+09$ & $3.30 \mathrm{E}+09$ & \\
${ }^{4} \mathrm{H}$ & $3.63 \mathrm{E}+18$ & $4.74 \mathrm{E}+18$ & \\
${ }^{6} \mathrm{He}$ & $7.12 \mathrm{E}+25$ & $5.46 \mathrm{E}+25$ & \\
${ }^{8} \mathrm{He}$ & $3.09 \mathrm{E}+33$ & $3.76 \mathrm{E}+32$ & \\
${ }^{7} \mathrm{Li}$ & $1.54 \mathrm{E}+32$ & $2.52 \mathrm{E}+32$ & \\
${ }^{8} \mathrm{Li}$ & $2.66 \mathrm{E}+36$ & $1.80 \mathrm{E}+36$ & \\
${ }^{9} \mathrm{Li}$ & $1.44 \mathrm{E}+41$ & $3.18 \mathrm{E}+40$ & \\
${ }^{11} \mathrm{Li}$ & $5.88 \mathrm{E}+46$ & $7.87 \mathrm{E}+46$ & \\
${ }^{7} \mathrm{Be}$ & $1.41 \mathrm{E}+34$ & $2.33 \mathrm{E}+32$ & $*$ \\
${ }^{9} \mathrm{Be}$ & $2.34 \mathrm{E}+44$ & $2.76 \mathrm{E}+44$ & \\
${ }^{10} \mathrm{Be}$ & $6.72 \mathrm{E}+49$ & $2.20 \mathrm{E}+49$ & \\
${ }^{11} \mathrm{Be}$ & $2.37 \mathrm{E}+53$ & $1.46 \mathrm{E}+53$ & \\
${ }^{12} \mathrm{Be}$ & $3.08 \mathrm{E}+57$ & $1.14 \mathrm{E}+57$ & \\
${ }^{14} \mathrm{Be}$ & $2.24 \mathrm{E}+63$ & $1.68 \mathrm{E}+63$ & \\
${ }^{10} \mathrm{~B}$ & $1.34 \mathrm{E}+50$ & $1.08 \mathrm{E}+50$ & $*$ \\
${ }^{11} \mathrm{~B}$ & $1.97 \mathrm{E}+56$ & $2.65 \mathrm{E}+56$ & \\
${ }^{12} \mathrm{~B}$ & $3.37 \mathrm{E}+60$ & $6.49 \mathrm{E}+60$ & \\
${ }^{14} \mathrm{~B}$ & $3.30 \mathrm{E}+68$ & $1.34 \mathrm{E}+69$ & \\
${ }^{15} \mathrm{~B}$ & $3.21 \mathrm{E}+72$ & $4.22 \mathrm{E}+72$ & \\
${ }^{16} \mathrm{C}$ & $9.20 \mathrm{E}+77$ & $2.70 \mathrm{E}+78$ & \\
${ }^{17} \mathrm{~B}$ & $5.26 \mathrm{E}+79$ & $2.05 \mathrm{E}+79$ & \\
${ }^{19} \mathrm{C}$ & 19 & & \\
${ }^{19} \mathrm{C}$ & $3.82 \mathrm{E}+82$ & $4.87 \mathrm{E}+82$ & \\
\end{tabular}

TABLE IV(a): Experimental [26,27] and calculated equilibrium constants for light isotopes observed in the ternary fission of ${ }^{242} \mathrm{Pu}$. Assigned upper experimental limits are indicated by ${ }^{* *}$. See text for details.

possible in the calculation.

\section{ACKNOWLEDGEMENTS}

This work was supported by the United States Department of Energy under Grant \# DE-FG0393ER40773, by the German Research Foundation (DFG), Grant \# RO905/38-1, the FCT (Portugal) Projects No. UID/FIS/04564/2019 and UID/FIS/04564/2020, and POCI-01-0145-FEDER-029912, and by PHAROS COST Action CA16214. H. P. acknowledges the grant CEECIND/03092/2017 (FCT, Portugal).

\section{APPENDIX A}

Tables $\mathrm{IV}(\mathrm{a})$ and $\mathrm{IV}(\mathrm{b})$ contain the $Q_{c}^{\text {eff }}$ values presented in Figure 1 of this paper. We note that $Q_{c}$ val-

\begin{tabular}{|c|c|c|c|}
\hline isotope & $Q_{c}^{\text {eff }}$ expt. & $Q_{c}^{\mathrm{eff}}$ calc. & upper limit \\
\hline${ }^{15} \mathrm{~N}$ & $2.89 \mathrm{E}+79$ & $8.24 \mathrm{E}+80$ & \multirow[t]{7}{*}{$* *$} \\
\hline${ }^{16} \mathrm{~N}$ & $1.42 \mathrm{E}+84$ & $3.26 \mathrm{E}+85$ & \\
\hline${ }^{17} \mathrm{~N}$ & $1.68 \mathrm{E}+89$ & $1.92 \mathrm{E}+90$ & \\
\hline${ }^{18} \mathrm{~N}$ & $2.17 \mathrm{E}+93$ & $2.74 \mathrm{E}+94$ & \\
\hline${ }^{19} \mathrm{~N}$ & $9.68 \mathrm{E}+97$ & $2.56 \mathrm{E}+98$ & \\
\hline${ }^{20} \mathrm{~N}$ & $2.96 \mathrm{E}+100$ & $1.22 \mathrm{E}+103$ & \\
\hline${ }^{21} \mathrm{~N}$ & $7.01 \mathrm{E}+104$ & $1.77 \mathrm{E}+107$ & \\
\hline${ }^{15} \mathrm{O}$ & $2.41 \mathrm{E}+83$ & $5.06 \mathrm{E}+79$ & \multirow[t]{5}{*}{$* *$} \\
\hline${ }^{19} \mathrm{O}$ & $2.97 \mathrm{E}+101$ & $8.11 \mathrm{E}+102$ & \\
\hline${ }^{20} \mathrm{O}$ & $3.45 \mathrm{E}+106$ & $4.22 \mathrm{E}+107$ & \\
\hline${ }^{21} \mathrm{O}$ & $1.98 \mathrm{E}+110$ & $3.20 \mathrm{E}+112$ & \\
\hline${ }^{22} \mathrm{O}$ & $2.83 \mathrm{E}+114$ & $1.14 \mathrm{E}+117$ & \\
\hline${ }^{24} \mathrm{O}$ & $1.42 \mathrm{E}+123$ & $1.77 \mathrm{E}+125$ & \multirow[t]{2}{*}{$* *$} \\
\hline${ }^{19} \mathrm{~F}$ & $7.00 \mathrm{E}+103$ & $1.37 \mathrm{E}+104$ & \\
\hline${ }^{20} \mathrm{~F}$ & $1.92 \mathrm{E}+107$ & $4.74 \mathrm{E}+109$ & \multirow[t]{5}{*}{$* *$} \\
\hline${ }^{21} \mathrm{~F}$ & $5.54 \mathrm{E}+112$ & $1.09 \mathrm{E}+115$ & \\
\hline${ }^{22} \mathrm{~F}$ & $2.17 \mathrm{E}+118$ & $1.31 \mathrm{E}+120$ & \\
\hline${ }^{24} \mathrm{~F}$ & $1.64 \mathrm{E}+126$ & $1.88 \mathrm{E}+129$ & \\
\hline${ }^{24} \mathrm{Ne}$ & $6.69 \mathrm{E}+129$ & $7.84 \mathrm{E}+132$ & \\
\hline${ }^{27} \mathrm{Ne}$ & $2.77 \mathrm{E}+142$ & $8.46 \mathrm{E}+145$ & $* *$ \\
\hline${ }^{24} \mathrm{Na}$ & $5.13 \mathrm{E}+131$ & $3.36 \mathrm{E}+134$ & $* *$ \\
\hline${ }^{27} \mathrm{Na}$ & $5.30 \mathrm{E}+145$ & $4.68 \mathrm{E}+149$ & \multirow[t]{2}{*}{$* *$} \\
\hline${ }^{28} \mathrm{Na}$ & $1.75 \mathrm{E}+150$ & $1.14 \mathrm{E}+154$ & \\
\hline${ }^{30} \mathrm{Na}$ & $1.75 \mathrm{E}+158$ & $3.94 \mathrm{E}+162$ & $* *$ \\
\hline${ }^{27} \mathrm{Mg}$ & $3.90 \mathrm{E}+148$ & $2.19 \mathrm{E}+152$ & $* *$ \\
\hline${ }^{28} \mathrm{Mg}$ & $1.61 \mathrm{E}+154$ & $4.45 \mathrm{E}+157$ & \multirow[t]{2}{*}{$* *$} \\
\hline${ }^{30} \mathrm{Mg}$ & $6.05 \mathrm{E}+162$ & $1.66 \mathrm{E}+167$ & \\
\hline${ }^{30} \mathrm{Al}$ & $4.12 \mathrm{E}+165$ & $5.20 \mathrm{E}+169$ & $* *$ \\
\hline${ }^{34} \mathrm{Si}$ & $3.94 \mathrm{E}+186$ & $1.53 \mathrm{E}+192$ & $* *$ \\
\hline${ }^{35} \mathrm{Si}$ & $2.95 \mathrm{E}+191$ & $6.67 \mathrm{E}+196$ & $* *$ \\
\hline${ }^{36} \mathrm{Si}$ & $1.08 \mathrm{E}+196$ & $2.12 \mathrm{E}+201$ & $* *$ \\
\hline
\end{tabular}

TABLE IV(b): Continued: Experimental [26,27] and calculated equilibrium constants for light isotopes observed in the ternary fission of ${ }^{242} \mathrm{Pu}$. Assigned upper limits are indicated by $* *$. See text for details.

ues far above the calculated values are derived for the isotopes ${ }^{7} \mathrm{Be}$ (parameter value 31 ) and ${ }^{15} \mathrm{O}$ (parameter value 71 ). Both values are based upon assigned upper limits. This comparison suggests that the actual yields for those two isotopes are well below these assigned values for upper limits. 
[1] M. Oertel et al., Rev. Mod. Phys.89, 015007 (2017).

[2] J. M. Lattimer and F. Douglas Swesty, Nucl. Phys. A 535, 331 (1991).

[3] H. Shen, H. Toki, K. Oyamatsu, K. Sumiyoshi, Nucl. Phys. A637, 435 (1998); Prog. Theor. Phys. 100, 1013 (1998).

[4] C. J. Horowitz and A. Schwenk, Phys. Lett. B638, 153 (2006); Nucl. Phys. A 776, 55 (2006).

[5] S. Typel et al., Phys. Rev. C 81, 015803 (2010).

[6] M. Hempel and J. Schaffner-Bielich, Nucl. Phys. A837, 210 (2010).

[7] H. Shen, H. Toki, K. Oyamatsu, and K. Sumiyoshi, Astrophys. J. Suppl. 197, 20 (2011).

[8] G. Shen, C. J. Horowitz, and E. O'Connor, Phys. Rev. C 83, 065808 (2011).

[9] M. D. Voskresenskaya and S. Typel, Nucl. Phys. A887, $42(2012)$.

[10] G. Röpke et al., Nucl. Phys. A897,70 (2013).

[11] S. Furusawa et al., Astrophys. J. 772, 95 (2013).

[12] B. G. Todd-Rutel and J. Piekarewicz, Phys. Rev. Lett. 95, 122501 (2005).

[13] H. Pais et al., Phys. Rev. C 97, 045805 (2018).

[14] H. Pais et al., Phys. Rev. Lett. 125, 012701 (2020).

[15] Dynamics and Thermodynamics with Nuclear Degrees of Freedom, Euro. Phys. J. 30, Issue 1. (2006) P. Chomaz et al. editors.

[16] "Topical issue on Nuclear Symmetry Energy." Eur. Phys. J. A 50 (2014). Bao-An Li et al., editors.

[17] J. Elliott et al., arXiv:1203.5132 (2012).

[18] L. Qin et al., Phys. Rev. Lett. 108, 172701(2012).

[19] Matthias Hempel et al., Phys. Rev. C91, 045805 ( 2015).

[20] C. Wagemans, The Nuclear Fission Process (CRC Press, Boca Raton, 1991). and references therein.

[21] J. Mills, Fission Product Yield Evaluation, Thesis Univ. of Birmingham (1995).

[22] IAEA-TECDOC-1168 ISSN 10114289 IAEA, Vienna, (2000). and references therein.

[23] I. Halpern, Ann. Rev. Nucl. Sci 21, 245 (1971).

[24] J. P. Theobald, Nucl. Phys. A502 343 (1989).

[25] G. K. Mehta et al., Phys. Rev. C 7, 373 (1973).

[26] O. Serot and C. Wagemans, Nucl. Phys. A641, 34 (1998).

[27] P. Heeg et al., Nucl. Inst. \& Meth. A278, 452 (1989).

[28] U. Koester Dissertation, Technischen Universität München (2000).

[29] U. Koester et al., Nucl. Phys. A652, 371 (1999).

[30] J. P. Lestone LA-UR-05-8860 (2006), Phys. Rev. C 72, 014604 (2005).

[31] I. Tsekhanovich, et al., Phys. Rev. C 67, 034610 (2003).

[32] R. Schafer and T. Fliessbach, J. Phys. G 21, 861 (1995).

[33] V. Rubchenya and S.Z. Yavshits, Z. Phys. A329, 217
(1988)

[34] V. A. Rubchenya Yad. Fiz. 35, 576 (1982).

[35] Yu. N. Kopatch et al., Phys. Rev. C 65, 044614 (2002).

[36] A. S. Vorobyev et al., J. Expt. And Theor. Phys. 127, 659 (2018).

[37] F. Goennenwein, M. Mutterer and Y. Kopatch, Europhys. News, 36, 11 (2005).

[38] S. Vermote et al., Seminar on Fission : Het Pand, Gent, Belgium, 17-20 May 2010 / editors, Cyriel Wagemans, Jan Wagemans, Pierre D'hondt. World Scientific Page 145 .

[39] E. Piasecki et al., Nucl. Phys. A255, 387 (1975).

[40] E. Piasecki and L. Nowicki, Proc. Symp. Phys. and Chem. Of Fission (Juelich 1979) IAEA, Vienna (1979) p 193.

[41] M. Sharma, PhD Thesis, Indian Institute of Technology, (1983).

[42] A. V. Ramayya, J.H. Hamilton and J.K. Hwang, Rom. Rept. Phys. 59, 595 (2007).

[43] A. Schubert,J. Hutsch and K. Müller, Z. Phys. A Hadrons and Nuclei 341, 481-488 (1992).

[44] C. Wagemans et al., Phys. Rev. C 78, 064616 ( 2008 ). Europhys. News. Jan./Feb. (2005).

[45] S. Wuenschel et al., Phys. Rev. C90, 011601(R) (2014).

[46] G. Röpke, J. B. Natowitz and H. Pais, submitted to Eur. J. Phys. A, August 2020.

[47] P. Demo and Z. Kozisek, J. Phys. G 23, 971 (1997).

[48] J. Schmelzer and G. Röpke, Phys. Rev. C 55, 1917 (1997).

[49] G. Wilemski and B.E. Wyslouzil, J. Chem. Phys. 103, 1127 (1995).

[50] A. Z. Mekjian, Phys. Rev. C 17, 1051 (1978).

[51] K. Hagel et al., Phys. Rev. C 62, 034607 (2000).

[52] M. Andronenko, et al., Eur. Phys. Jour. A 12, 185 (2001).

[53] M. Popkiewicz et al. (1996), https://www.osti.gov/etdeweb/servlets/purl/583067.

[54] L. G. Sobotka et al., Phys. Rev. C 55, (1997).

[55] K. Hagel et al., Phys. Rev. Lett. 108, 062702 (2012).

[56] I. Cervesato et al., Phys. Rev. C 45, 2369 (1992).

[57] BNL ENSDFENSDF Evaluated Nuclear Structure Data File. https://www.nndc.bnl.gov/].

[58] G. Röpke, L. Münchow and H. Schulz, Nucl. Phys. A379, 536 (1982).

[59] W. von Oertzen, Int. J. Mod. Phys. E, 20, 765 (2011).

[60] K. Kisamori, et al., Phys. Rev. Lett. 116, 052501 (2016).

[61] O. Ivanytskyi, M. Angeles Perez-Garcia, and C. Albertus, Eur. Phys. J. A 55184 (2019).

[62] G. Röpke, Phys. Rev. C 101, 064310 (2020).

[63] G. Röpke et. al. paper in preparation. 